\title{
IS IT APPROPRIATE TO USE THE OBJECTIVE, RATIONAL DECISION-MAKING FRAMEWORK AS A FOUNDATION FOR MODELLING SOCIAL SYSTEMS?
}

\author{
Marko A. HOFMANN and Heiko HAHN
}

\begin{abstract}
In general, the developers of Decision Support Systems (DSS) look for objectivity and the support of the rational choice. These guiding principles are rarely reflected upon because they are so successful for technical problems and within the humanities. However, the majority of DSS for military purposes-especially in the context of the new tasks related to transformation of the armed forces - have to deal with social systems. These systems differ significantly from systems with no human interaction. First, there is an irreducible amount of uncertainty about the cause-effect-relations in such systems, which leads to unpredictability of many phenomena. Second, there is also a non-negligible amount of uniqueness in each event that makes it extremely hard to find general rules, which may help to find reproducible behavior. Third, most events cannot be evaluated from an objective point of view since every evaluation depends on subjective criteria. In the quest for objectivity and using the Rational Analytical Decision-Making Framework (RADMF) easily leads to erroneously take personal assumptions for apodictic postulations, which are originally based on prejudice and dogmatism. It also often results in lack of critical discussion because an objectively-best solution can only be questioned outside the decision framework. Therefore, this article advises on the use of transparency instead of objectivity and of heuristic decision frameworks instead of the RADMF.
\end{abstract}

Keywords: Social Systems, Rational-Analytic Decision Making Framework, Objectivity, Transparency, Heuristics.

\section{Introduction}

One of the main objectives of Operations Research since the first days of this science has been the development of objective, rational decision-making frameworks. The promotion of such frameworks is often taken for granted. However, the concept of objectivity and the methodology of rational-decision making are not unshakeable pillars of modeling and simulation. The limitations of both, the concept and the meth- 
odology, are highlighted within social simulations and other models of human interaction systems.

This article focuses on decision-making in concrete, critical, complex and controversial situations ( $\mathrm{C}^{4}$-situations), which means that the problem that has to be solved is:

- A currently unsolved real-world problem with no academic simplifications or generalizing abstractions (concrete);

- $\quad$ Time-critical (in the sense that the time available for decision-making is limited with respect to accessible, problem-relevant data, and also in the sense that the time horizon for which the results of the decision have to be evaluated is $a$ priori unclear and, therefore, likely to be underestimated) and value critical (personal danger, damage, costs, etc.) (critical);

- Situated in an environment with nonlinear relationships and different types of feedback loops between causes and effects (complex);

- Differently perceived by at least two opposing social groups, with, at least, incompatible goals (controversial).

Also, it is assumed that the decision-making process is based on the evaluation of possible consequences of different available options.

\section{Objectivity}

\section{General Remarks}

From the perspective of philosophy of science, objectivity is an extremely controversial concept and if it is used at all, it is based on reproducible, observer-independent, quantified (with commonly accepted measures) empirical data. Whereas in natural science experiments can be designed that produce that data quality, the observations in social systems can seldom claim to be scientifically reproducible, observer-independent and quantified in a commonly accepted manner. As a simple example, consider observations of human behavior in contemporary Baghdad. There is an irreducible amount of uniqueness in every assault, in every demonstration, in the response to every military action, resisting all attempts to make them reproducible. The states of mind of the Iraqi people can hardly be evaluated in an observer-independent manner and the quantitative everyday statistics of losses hardly captures the essence of the violence. Beside these practical problems, we simply do not know enough about such systems in order to claim objectivity: Every modeler has to make a plethora of subjective assumptions about human factors like motivation or fear, the interaction of causes and effects, the adequate borders of the reference system and much more. The strife for objectivity in modeling such hardly understood social systems can easily lead to erroneously accept personal assumptions for apodictic postulations based on prejudice and dogmatism. Every model of social processes can only be one 
possible, subjective "representation" of an original process, in which even truth is a volatile concept.

\section{A Philosophical Inquiry}

"Objectivity" is a concept that is only applicable in epistemologies based on realism. It is almost irrelevant in others. Thus, it is a matter of one's philosophical attitude whether objectivity is possible or not. Epistemology is a branch of philosophy, dealing with gaining and justification of knowledge. It attempts to answer the fundamental question: What distinguishes true/ adequate knowledge from false/ inadequate knowledge? Epistemological considerations almost directly translate into the philosophy of science. In the context of model validity, epistemological questions set the frontiers of what can be known by modeling and simulation. Unfortunately, there is no single prevailing epistemological perspective in philosophy. Here, it would go much too far to discuss all positions in the philosophy of science. Fortunately, the point we want to make can also be discussed based on a coarse distinction between the two main classes of epistemology: objectivism and relativism, from which we take two rather extreme proxies (which are by no means representative for all positions but highlight the essential differences) - positivism and constructivism.

The positivistic paradigm for gaining scientific knowledge is based on the belief that reality is independent from the human observer's perception and is totally governed by laws of nature (naive-realistic ontology). The positivistic epistemology is founded on the notion that humans can fully "understand" reality and that experiments can reveal the "true" (in the sense of "observer independent") nature of a phenomenon. A human observer is seen as an instance of a stimulus-reaction mechanism. The methodology is completely constrained to empiric experimentalism - all open questions are formulated as hypotheses, which are corroborated or refuted on the basis of experiments. Knowledge is consequently the correspondence between reality and the mental or formal representation (correspondence theory of truth). Today, in practice, this position is often attenuated to a kind of "pragmatic realism," which means that scientists have the aim of developing and using models that are as "realistic as possible," given the constraints of current knowledge, skills, computing power and available time.

In contrast, the ontological foundation of constructivism is based on idealism -different subjective realities coexist as mental constructs. The corresponding epistemological position is rationalism. The observer and his or her cognitive apparatus are not neutral. In the process of perception, the cognitive system functions as a synthesizing agent based upon the cognitive state and the inputs from the environment. The "raw data" is never perceived raw but always as already interpreted. With other words, each observation is the result of an interaction between observer and observed situa- 
tion, thus the results are strongly influenced by the observer's knowledge, attitudes and values. To a certain extent, humans are the creators of their own reality. The most important methodological technique of the constructivist paradigm is interpretation. The goal of knowledge seeking in constructivism is not absolute "truth" but relative "viability." A mental construct of reality is said to be viable if it helps a subject in its struggle for life.

According to the constructivist view, the validation of simulation results against empirical data sets is not about comparing the real world and the simulation output; it is comparing what you observe as real world with what you observe as output. Both are constructions of an observer and his/her views concerning relevant agents and their attributes. Constructing reality and constructing simulation are just two ways of an observer seeing the world.

Clearly, such an observer-oriented view of the world is unsatisfying to most scientists and in order to avoid both solipsism and indiscriminate relativism it is indeed necessary to explain how individual perceptions and constructions of the world converge to common pictures of reality that are shared and trusted within scientific communities. Ultimately, this convergence is nothing more than a consensus about the reality observed by the members of a special community. This consensus is generated on the grounds of ordinary everyday social interaction that creates an area of shared meanings and expectations. Thus, science (especially the meaning of scientific results) is established on the basis of discussed and confirmed observations both on reality and model. In the case of practical decision-making, this discussion must be chaired by the decision makers themselves. However, in order to be discussed, the models have to be understandable to non-experts, too. For that reason, the transparency of decision support models is of much greater importance than the unachievable objectivity.

\section{Pragmatic Aspects}

Besides all these philosophical considerations, there are also some difficult practical problems that limit the use of "objectivity" as a guideline in the design and use of decision-making frameworks for $\mathrm{C}^{4}$-situations:

- It is extremely hard to evaluate and foresee reactions of humans driven by religion or intensive emotion from a completely observer-independent point of view.

- The predictive validity of social models is notoriously low. One can even claim that any social model that shows a certain amount of predictive validity has to lose this attribute as soon as it is known to have it. Otherwise, we could easily build a "perpetual cash machine" at the stock market. 
- In general, a plethora of subjective assumptions is necessary to fill all kinds of information gaps. One reason, in fact, to build simulations is to explore phenomena for which no or only insufficient empirical data exists. The so called "data-poor environments" are the standard in sociology and often in military operations research, too. In most cases the lack of information is not a matter of effort, but a fundamental problem. In these applications, simulation is mainly an extended "thought experiment" done with the support of computers.

Hence, even from a mere pragmatic perspective, it is hardly possible to claim objectivity.

\section{Rational Decision Making}

\section{General Remarks}

A similar criticism is applicable to the method, the Rational Analytical Decision Making Framework (RADMF). Rational decision-making is a well-defined methodology based on the analytic comparison of different options within a so-called decision framework, which consists - in military context —of environmental system states including enemy options, probabilities $(p)$ of these environments, and quantitatively evaluated consequences of own options against enemy options based on a global objective function (see Table 1). The best decision is calculated by maximization of the expected utility value. ${ }^{1}$

None of these elements is certain for a social system in wartime. We hardly ever know completely our own options, can sometimes only guess about the enemy, have to make assumptions about future environments and their probabilities and have to interpret calculations or simulations of the outcomes with uttermost care. There is so much uncertainty inherent in almost every phenomenon affecting such decisions that trying to follow the rational decision-making process is often irrational and self-de-

Table 1: General Structure of the Rational Analytical Decision Making Framework.

\begin{tabular}{|c|c|c|c|c|}
\hline & Environment 1 & Environment 2 & Environment 3 & Environment 4 \\
\hline & $p_{1}$ & $p_{2}$ & $p_{3}$ & $p_{4}$ \\
\hline Option A & Consequence A-1 & Consequence A-2 & Consequence A-3 & Consequence A-4 \\
\hline Option B & Consequence B-1 & Consequence B-2 & Consequence B-3 & Consequence B-4 \\
\hline Option C & Consequence C-1 & Consequence C-2 & Consequence C-3 & Consequence C-4 \\
\hline
\end{tabular}


ceiving. The Nobel laureate Herbert Simon used the term "bounded rationality" to indicate these limits of rationality. Modern psychology has confirmed his findings and underpinned the indispensable role of heuristic and intuitive decision-making. Thus, the essential criterion of a successful decision-making framework for social systems should not be formal rationality in the sense of the common RADMF. As a substitute, the authors propose to look for highly adaptive, more heuristic frameworks enabling the decision maker to access a broad range of cognitive functions.

\section{Principles of Bounded Rationality}

The difference between the RADMF and all approaches based on the notion of bounded rationality can be summarized using the following five main principles ${ }^{2}$ :

- Exploration of possible options replaces any attempt for complete evaluation of "all" options.

- With respect to the results of the decision-making process, precision is much less important than robustness due to data uncertainty.

- There are no absolute decisions, all of them are context-dependent and "moderate" (suitable versus unsuitable in contrast to right versus wrong).

- Objective optimization is replaced by subjective "satisficing."

- If possible, each "big" decision is replaced by a set (sequence) of small decisions and thereby ultimately postponed in order to avoid irreversible failures (an attitude, which is also called incrementalism).

These principles show that the methods of bounded rationality take uncertainty for a fact. $^{3}$

\section{Examples of Decision Making based on Bounded Rationality}

In what follows, the authors provide a brief description of three examples of nonanalytical decision-making in order to illustrate the principles of bounded rationality. These examples are: simple heuristics, assumption-based planning, and scenario planning. The descriptions are restricted to the essential features.

\section{Simple Heuristics}

The heuristic method is a problem-solving technique that ignores the fact whether it could be proved that the solution is correct, but which usually produces a good solution. ${ }^{4}$ Also, the heuristic approaches often ignore some of the available data. They also do not guarantee optimal solutions. In general, these methods are very fast and economical with respect to data requirements, and yield strikingly good results when information is scarce or uncertainty - high. 
According to the take-the-best heuristic, for example, when making a judgment based on multiple criteria, the criteria are tried one at a time according to their cue validity, and a decision is made based on the first criterion that discriminates between the alternatives. Gigerenzer and Selten ${ }^{5}$ discovered that the heuristic method did surprisingly well at making accurate inferences in real-world environments, such as inferring which of two cities is larger. In order to decide, you look at an ordered list of binary cues. First, is one of the cities the national capital? Second, is one a state capital? Third, does one of them have a major-league soccer team? And so on. The first time you get "yes" for one city and "no" for the other, guess that the "yes" city is bigger. Take-the-Best works very well if you have a good set of cues arranged in the right order. It is not perfect, however. The important thing is that this imperfection is wellknown to the decision-maker.

The heuristic approach has since been modified and applied successfully to domains from medicine to artificial intelligence.

\section{Assumption-Based Planning}

Assumption-based planning ${ }^{6}$ is a method that helps to deal with uncertainty. It is used to identify the most important (most critical) assumptions in a plan or idea, test these assumptions and think of hedging actions. A plan is regarded to be a tentative solution to the inexact problems posed by an uncertain future. Even if the future could be known with certainty (which is unlikely), there are likely to be uncertainties about whether a given plan is the proper solution or approach to that future. Any plan contains assumptions about what the future holds (the problems in the future) and whether the plan will perform as hoped (the solution to those problems).

Assumption-based planning is used best when a plan has already been constructed; the techniques are used to improve the robustness and adaptability of the plan by reducing the number of surprises that occur by decreasing the risks posed by assumptions made during the planning process.

Assumption-based planning (ABP) is performed in five basic steps ${ }^{7}$ :

1. List all assumptions in the plan.

2. Identify load-bearing vulnerable assumptions.

3. Create signposts.

4. Take shaping actions.

5. Take hedging actions.

According to the developers of ABP, a planning assumption is a judgment or evaluation about some characteristic of the future that underlies the plans of an organization. All planning requires some judgments about, or evaluations of, the future. The more 
uncertain the future, the more likely it is that the planner will have to make judgments or "guesstimates" to help narrow the range and number of uncertainties about the future into a manageable few before progress can be made. Dewar ${ }^{8}$ also provides a list of techniques to find assumptions in a plan.

The next step in $\mathrm{ABP}$ is to identify those assumptions upon which the success of the plan must rest - the so-called "load-bearing" assumptions - and the assumptions that are most vulnerable to being overturned by future events - assumptions which are likely to crumble. Assumptions that are both load-bearing and vulnerable are the most likely to produce dangerous surprises as the plan unfolds. To deal with potential surprises, ABP uses signposts, shaping actions, and hedging actions. Signposts are warning signs that can be used to monitor these assumptions that are most likely to produce surprises. Signposts are events or thresholds that, if detected, signify that a vulnerable assumption is broken or dangerously weak and that management or planning action is called for. Shaping actions are intended to help shore up uncertain assumptions; they are attempts to control the future as much as possible. Planners generally know how they would like an assumption to play out; hence shaping actions are designed to help the assumption play out to the planners' liking. Hedging actions, in contrast, better prepare for the possibility that an assumption will fail, despite efforts to shore it up. Hedging actions typically come from thinking through a plausible scenario in which an assumption collapses and asking what might be done now to prepare for that scenario.

\section{Scenario Planning}

A scenario describes a possible future environment, but is not a prediction. It combines known facts about the future with plausible trends, which are key driving forces. ${ }^{9}$ A scenario explores the extremes, which challenge the existing mental conception of the future. It should be engaging, interesting, challenging and credible, as well as logically consistent with the known facts. A set of scenarios describes a range of possible futures and should be mutually exclusive and collectively exhaustive. Ideally, the set should have no more than four scenarios or it becomes difficult to manage. Scenarios can be presented in many different forms, such as in a script, a timeline, within a discussion, or as a computer simulation. Scenario planning can include anticipatory thinking elements that are difficult to formalize, such as subjective interpretations of facts, shifts in values, new regulations, inventions and even intuitions and visions. However, the descriptive scenarios need to be supported by numerical analysis, which should:

- Test the credibility of each scenario;

- Explore the magnitude of changes in the environment;

- Evaluate the impact of those changes. 
The scenarios are then used to challenge existing conceptions of the future and stimulate new ideas. They form the basis of a (in general) strategic debate, which is drastically different to the traditional strategic planning cycle. In the best case, scenario planning creates a flexible plan which is composed of a variety of options, which might be seen as contingency plans. This enables the decision maker to adapt his/her plans to the evolving environment.

According to the leading developers of scenario planning Van der Heijden and coworkers, ${ }^{10}$ it is a process that

- Surfaces and challenges assumptions and mindsets in a constructive and creative way;

- Recognizes uncertainty and makes it explicit;

- Provides a way of making sense of an overabundance of information;

- Facilitates the development of new insights that would not reside it from conventional planning methods;

- Ensures that all of this is achieved as an organization, in which individuals align on coherent action;

- Integrates thinking, planning and key future investments to exploit the scarcity landscape.

In short, scenario planning allows organizations to rehearse the future, to walk the battlefield before the battle. The result is not, however, a more accurate prediction of the future, but the ability to better distinguish the significant aspects of the future. This, in turn, allows better decisions to be made.

\section{Summary of the Difference between Methods of Bounded Rationality and RADMF}

Comparing the RADMF and the methods of bounded rationality, some major differences come to mind. The latter

- Lead to results, which are ex-ante and ex-post only approximations;

- Take a critical stance towards the predictability of the future;

- Allow discussions about the reasoning itself, not only about the input data;

- Do not claim objectivity;

- Take unexpected events to be unavoidable;

- Easily accept adaptations of own decisions since nothing "has to be defended;"

- Do not only accept rationality, but also intuition. ${ }^{11}$ 
The main difference, however, is that the alternatives are inherently highly adaptive self-critical frameworks with respect to the current knowledge that take a significant amount of uncertainty as irreducible.

\section{Summary and Conclusion}

This article has argued that objectivity is hardly ever possible in decision-making affecting social systems. The two main reasons for that are a general lack of knowledge about the cause-effect relationships related to a given phenomenon and an irreducible amount of uniqueness in every single event within such "self-reflective" systems.

The authors believe that striving for objectivity makes it difficult to keep a skeptical/ critical attitude towards own decisions, since so much efforts are made to eliminate all disputable aspects of the reasoning. Such an endeavor can also easily lead to futile attempts to find corroborating empirical evidence, which can only be anecdotic because of the uniqueness problem.

The authors have further tried to illuminate why the rational analytical decision making framework is ill-suited for concrete, complex, time and value critical, controversial decisions with substantial uncertainty. The framework easily leads to disproportionate trust into formal aspects like completeness and optimality in environments where uncertainty and unpredictability (and therefore unexpected events) are and must be the key factors of all considerations. Due to the impossibility to make objectively-best solutions, decision-making in social systems has to be founded on subjective arguments. Decision support systems can help to avoid mere debating by making these arguments explicit within a common framework of discussion.

However, in order to allow such discussion, the focus of this support models must shift towards transparency, flexibility, and adaptability. The principles of bounded rationality can help to construct such frameworks. Concrete examples for such frameworks can be found studying assumption-based and scenario planning.

\section{Notes:}

${ }^{1}$ R. Duncan Luce and Howard Raiffa, Games and Decisions: Introduction and Critical Survey (New York: John Wiley \& Sons, 1957); Ralph L. Keeney and Howard Raiffa, Decisions with Multiple Objectives: Preferences and Value Trade-Offs (Cambridge University Press, 1993). 
2 Herbert A. Simon, Models of Bounded Rationality, Volumes 1-3 (Cambridge, MA: The MIT Press, 1982, 1997).

3 Gerd Gigerenzer, Adaptive Thinking: Rationality in the Real World (New York: Oxford University Press, 2000).

${ }^{4}$ Gerd Gigerenzer, Peter M. Todd, and the ABC Research Group, Simple Heuristics that Make Us Smart (New York: Oxford University Press, August 1999).

5 Gerd Gigerenzer and Reinhard Selten, eds., Bounded Rationality: The Adaptive Toolbox (Cambridge, MA: The MIT Press, February 2001).

6 James A. Dewar, Carl H. Builder, William M. Hix, and Morlie H. Levin, Assumption-Based Planning: A Planning Tool for Very Uncertain Times (Santa Monica: RAND Corporation, January 1993).

7 James A. Dewar, Assumption-Based Planning: A Tool for Reducing Avoidable Surprises (Cambridge, UK: Cambridge Press, 2002).

8 Dewar, Assumption-Based Planning: A Tool for Reducing Avoidable Surprises.

9 Kees van der Heijden, Ron Bradfield, George Burt, George Cairns, and George Wright, The Sixth Sense: Accelerating Organizational Learning with Scenarios (Chichester: John Wiley \& Sons, July 2002).

${ }^{10}$ Van der Heijden, Bradfield, Burt, Cairns, and Wright, The Sixth Sense: Accelerating Organizational Learning with Scenarios.

${ }^{11}$ Gerd Gigerenzer, Gut Feelings: The Intelligence of the Unconscious (New York: Viking Press, July 2007) (UK edition: Short Cuts: The Intelligence of the Unconscious (London: Penguin Books, 2007).

MARKO HOFMANN is Project Manager at the Institute for Technology of Intelligent Systems (ITIS) at the University of the Federal Armed Forces of Germany in Neubiberg. After his studies of Computer Science at the University of the Federal Armed Forces from 1990 to 1993, he served two years in an army battalion staff. From 1995 to 2000 he was research assistant at the Institute for Applied System Analysis and Operations Research (IASFOR), where he obtained his Ph.D. in Computer Science. Since April 2000 he has been responsible for basic research in applied computer science at the Institute for Technology of Intelligent Systems (ITIS). The main focus of his research is the modeling and simulation of complex systems in order to support decision making in critical situations. He gives lectures at the University of the Federal Armed Forces in Munich and at the University of Applied Science in Kufstein.

HEIKO HAHN received his Ph.D. in Management Information Systems (MIS) from the University of Augsburg, Germany. He worked as a post-doctoral fellow at the University of New Mexico for six months. Currently, he is a post-doctoral fellow at the faculty of Computer Science at the University of the Federal Armed Forces in Munich, Germany. His main research areas are statistical and experimental analysis in the field of Operations Research. 